\title{
Seshadri constants in finite subgroups of abelian surfaces.
}

\author{
Luis Fuentes García
}

Luis García López in memoriam.

\begin{abstract}
Given an étale quotient $q: X \longrightarrow Y$ of smooth projective varieties we relate the simple Seshadri constant of a line bundle $M$ on $Y$ with the multiple Seshadri constant of $q^{*} M$ in the points of the fiber. We apply this method to compute the Seshadri constant of polarized abelian surfaces in the points of a finite subgroup.

MSC (2000): Primary 14C20; secondary, 14E20.

Key Words: Étale quotients, multiple Seshadri constants, abelian surfaces.
\end{abstract}

\section{Introduction.}

The multiple Seshadri constants are a natural generalization of the Seshadri constants at single points defined by Demailly in [5]. If $X$ is a smooth projective variety of dimension $n, L$ is an ample line bundle on $X$ and $x_{1}, \ldots, x_{r}$ are distinct points in $X$, then the Seshadri constant of $L$ at $x_{1}, \ldots, x_{r}$ is:

$$
\epsilon\left(L ; x_{1}, \ldots, x_{r}\right)=\sup \left\{\epsilon \mid f^{*} L-\epsilon \sum_{i=1}^{r} E_{i} \text { is nef }\right\},
$$

where $f$ is the blowing up of $X$ at $x_{1}, \ldots, x_{r}$ and $E_{1}, \ldots, E_{r}$ are the exceptional divisors. These constants have the upper bound:

$$
\epsilon(L ; r) \leq \sqrt[n]{\frac{L^{n}}{r}}
$$

However, explicit values are difficult to obtain even when $r=1$. General bounds for the simple Seshadri constants on surfaces are given in [2], 7] or 9]. They were computed for simple abelian surfaces by Th. Bauer (see [2]); Ch. Schultz gave values for Seshadri constants on products of two elliptic curves (see [8]).

The case of the multiple Seshadri constants is harder. For example, in the plane the Nagata conjecture is still an open problem (see [10]): 
Conjecture 1.1 (Nagata conjecture) Let $x_{1}, \ldots, x_{r}$ be $r \geq 10$ be general points in $P^{2}$ then:

$$
\epsilon\left(\mathcal{O}_{P^{2}}(1) ; x_{1}, \ldots, x_{r}\right)=\frac{1}{\sqrt{r}}
$$

This has been extended for an arbitrary surface. When $r$ is big enough, the value of the Seshadri constant at $r$ very general points is conjectured to be maximal (see [4). Very intersting lower bounds for multiple Seshadri constants were given by B. Harbourne in [6]. In [1] Tutaj-Gasińska give bounds for the Seshadri constant of abelian surfaces in half-periods points; in [12, he gives the exact values in two half-periods points.

In this paper, we obtain the exact value of the multiple Seshadri constants of polarized abelian surfaces in points of a finite subgroup. This generalizes the results of [11] and [12] but applying a different method.

If $q: X \longrightarrow Y$ is an étale quotient of smooth projective varieties we prove that the simple Seshadri constant of a line bundle $M$ on $Y$ is the same that the multiple Seshadri constant of $q^{*} M$ in the points of the fiber. We apply this result when $X$ is an abelian surface and $Y=X / G$ is the quotient by a finite subgroup $G$. Since, the simple Seshadri constants on abelian surfaces are known (see [2], [8]), we obtain the multiple Seshadri constants on $G$.

In particular, when $X$ is an abelian surface with Picard number one, we prove the following:

Theorem 1.2 Let $(X, L)$ be a polarized abelian surface of type $(1, d)$ with $\rho(X)=$ 1. Let $x$ be a point of $X$. Let $G$ be a finite subgroup of $X$ of order $g$. Consider the étale quotient:

$$
q: X \longrightarrow X / G
$$

Let $n$ be the minor integer verifying $n L=q^{*} M$ for some line bundle $M$ on $X / G$. Then:

1. If $\sqrt{2 d / g}$ is rational, then $\epsilon(L ; x+G)=\sqrt{\frac{2 d}{g}}$.

2. If $\sqrt{2 d / g}$ is irrational, then

$$
\epsilon(L ; x+G)=\frac{k_{0}}{l_{0}} \frac{2 d n}{g}=\sqrt{1-\frac{1}{l_{0}^{2}}} \sqrt{\frac{L^{2}}{g}}
$$

where $\left(l_{0}, k_{0}\right)$ is the primitive solution of Pell's equation $l^{2}-\frac{2 n^{2} d}{g} k^{2}=1$.

Note, that the order of $G$ and the degree of $L$ is not sufficient to determine the value of the Seshadri constants. It depends also on the the structure of $G$ (see Remark 3.4 for details).

In general, we also prove: 
Theorem 1.3 Let $(X, L)$ be a polarized abelian surface. The multiple Seshadri constant of $L$ at the points of a finite subgroup is rational.

\section{The main theorem.}

Theorem 2.1 Let $q: X \longrightarrow Y$ be an étale $n: 1$ quotient between to smooth projective varieties. Let $M$ be a line bundle on $Y$ and $y \in Y$. Then:

$$
\epsilon\left(q^{*} M ; q^{-1}(y)\right)=\epsilon(M, y)
$$

Proof: Let $g: \tilde{Y} \longrightarrow Y$ be the blowing up of $Y$ at $y$. Let $f: \tilde{X} \longrightarrow X$ be the blowing up of $X$ at $q^{-1}(y)$. There is an induced morphism $\tilde{q}: \tilde{X} \longrightarrow \tilde{Y}$ such that the following diagram is commutative:

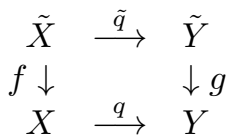

If $E$ is the exceptional divisor of $g$ and $E_{1}, \ldots, E_{n}$ are the exceptional divisors of $f$, we have:

$$
f^{*} q^{*} M-\epsilon\left(E_{1}+\ldots+E_{n}\right)=\tilde{q}^{*} g^{*} M-\epsilon \tilde{q}^{*} E=\tilde{q}^{*}\left(g^{*} M-\epsilon E\right) .
$$

Thus,

$$
f^{*} q^{*} M-\epsilon\left(E_{1}+\ldots+E_{n}\right) \text { is nef } \Longleftrightarrow g^{*} M-\epsilon E \text { is nef. }
$$

\section{Multiple Seshadri constant on polarized abe- lian surfaces.}

We will apply the main theorem to compute the multiple Seshadri constants on polarized abelian surfaces. We will use the results of Bauer to compute the simple Seshadri constant (see [2]):

Theorem 3.1 (Bauer) Let $(Y, M)$ be an abelian surface of type $\left(1, d^{\prime}\right)$ with $\rho(Y)=1$,

1. If $\sqrt{2 d^{\prime}}$ is rational, then $\epsilon(M)=\sqrt{2 d^{\prime}}$.

2. If $\sqrt{2 d^{\prime}}$ is irrational, then

$$
\epsilon(M)=\frac{k_{0}}{l_{0}} 2 d^{\prime}=\sqrt{1-\frac{1}{l_{0}^{2}}} \sqrt{M^{2}}
$$

where $\left(k_{0}, l_{0}\right)$ is the primitive equation of the Pell's equation $l^{2}-2 d k^{2}=1$. 
Let us recall some basic facts about abelian surfaces. We will follow the notation of $[3$. Let $(X, L)$ be a polarized abelian surface of type $(1, d)$. Let $H$ be the first Chern class of $L$. There is a basis $\lambda_{1}, \lambda_{2}, \mu_{1}, \mu_{2}$, respect to which $E=\operatorname{Im} H$ is given by the matrix:

$$
\left(\begin{array}{rr}
0 & D \\
-D & 0
\end{array}\right)
$$

where $D=\operatorname{diag}(1, d)$. In this way, the abelian surface $X$ is the quotient

$$
\pi: V \longrightarrow X=V / \Lambda
$$

with

$$
\Lambda=\left\langle\lambda_{1}, \lambda_{2}\right\rangle \oplus\left\langle\mu_{1}, \mu_{2}\right\rangle .
$$

Let $G$ be a finite subgroup of $X$ of order $g$. Consider the quotient map:

$$
q: X \longrightarrow X / G \text {. }
$$

The variety $Y=X / G$ is an abelian surface. In particular $Y=V / \Lambda^{\prime}$, where $\Lambda^{\prime}=\pi^{-1}(G)$. There is a criterion for a line bundle $L^{\prime} \in \operatorname{Pic}(X)$ to descend under $q$ :

Lemma 3.2 Let $L^{\prime}=L\left(H^{\prime}, \chi^{\prime}\right)$ be a line bundle on $X$. Then, $L^{\prime}=q^{*} M$ for some line bundle $M \in \operatorname{Pic}(Y)$ if and only if $\operatorname{Im}^{\prime}\left(\Lambda^{\prime}, \Lambda^{\prime}\right) \subset \mathrm{Z}$.

Proof: See Chapter 2, Corollary 4.4 of [3].

Corollary 3.3 If $L$ is a line bundle on $X$ of type $(1, d)$, then $\exp (G)^{2} L=q^{*} M$ for some $M \in \operatorname{Pic}(Y)$.

Proof: It is a consequence of the previous corollary. The exponent of a group $G$ is the least common multiple of the orders of the elements of $G$. The first Chern class of $\exp (G)^{2} L$ is $\exp (G)^{2} H$. Let $x, x^{\prime} \in \pi^{-1}(G)$. We know that $\exp (G) \cdot x, \exp (G) \cdot x^{\prime} \in \Lambda$. Thus:

$$
\operatorname{Im}\left(\exp (G)^{2} H\right)\left(x, x^{\prime}\right)=\operatorname{Im} H\left(\exp (G) \cdot x, \exp (G) \cdot x^{\prime}\right) \in \mathrm{Z} .
$$

Remark 3.4 We can consider the minor integer $n$ such that $n L$ descend to a line bundle $M$. The minimality of $n$ implies that $M$ is a primitive line bundle on $Y$. We know that $1 \leq n \leq \exp (G)^{2}$. However, the number $n$ does not depend only on the exponent of $G$. For example, if $G$ is the cyclic group generated by $\pi\left(\lambda_{2} / d\right)$ then $L$ descends to a line bundle $M$ of type $(1,1)$, so $n=1$. On the other hand, if $G$ is the group generated by $\pi\left(\lambda_{1} / k\right), \pi\left(\mu_{1} / k\right)$ for any $k>1$ then the minor value of $n$ is $n=k^{2}=\exp (G)^{2}$. 
The main result on abelian surfaces with Picard number one will be the following:

Theorem 3.5 Let $(X, L)$ be a polarized abelian surface of type $(1, d)$ with $\rho(X)=$ 1. Let $x$ be a point of $X$. Let $G$ be a finite subgroup of $X$ of order $g$. Consider the étale quotient:

$$
q: X \longrightarrow X / G
$$

Let $n$ be the minor integer verifying $n L=q^{*} M$ for some line bundle $M$ on $X / G$. Then:

1. If $\sqrt{2 d / g}$ is rational, then $\epsilon(L ; x+G)=\sqrt{\frac{2 d}{g}}$.

2. If $\sqrt{2 d / g}$ is irrational, then

$$
\epsilon(L ; x+G)=\frac{k_{0}}{l_{0}} \frac{2 d n}{g}=\sqrt{1-\frac{1}{l_{0}^{2}}} \sqrt{\frac{L^{2}}{g}}
$$

where $\left(l_{0}, k_{0}\right)$ is the primitive solution of Pell's equation $l^{2}-\frac{2 n^{2} d}{g} k^{2}=1$.

Proof: Let $y=q(x)$. By the Theorem 2.1

$$
\epsilon(L ; x+G)=\frac{1}{n} \epsilon(n L ; x+G)=\frac{1}{n} \epsilon(M, y) .
$$

The line bundle $M$ is a primitive line bundle of type $\left(1, d^{\prime}\right)$ with $d^{\prime}=n^{2} d / g$. Thus $\sqrt{2 d / g}$ is rational if and only if $\sqrt{2 d^{\prime}}$ is rational. Now, the result follows from Theorem 3.1

Corollary 3.6 Let $(X, L)$ be a polarized abelian surface of type $(1, d)$ with $\rho(X)=1$. Let $x_{1}, \ldots, x_{r}$ be $r$ general points of $X$. Then:

1. If $\sqrt{2 d / r}$ is rational, then $\epsilon\left(L ; x_{1}, \ldots, x_{r}\right)=\sqrt{\frac{2 d}{r}}$.

2. If $\sqrt{2 d / r}$ is irrational, then

$$
\epsilon\left(L ; x_{1}, \ldots, x_{r}\right) \geq 2 d \frac{k_{0}}{l_{0}}=\sqrt{1-\frac{1}{l_{0}^{2}}} \sqrt{\frac{L^{2}}{r}}
$$

where $\left(l_{0}, k_{0}\right)$ is the primitive solution of Pell's equation $l^{2}-2 r d k^{2}=1$.

Proof: By the semicontinuity of the Seshadri constant,

$$
\epsilon\left(L ; x_{1}, \ldots, x_{r}\right) \geq \epsilon(L ; x+G)
$$

for any point $x \in X$ and any subgroup $G$ of order $r$. In particular, taking the cyclic subgroup $G=\left\langle\pi\left(\lambda_{1} / r\right)\right\rangle$ and applying the previous theorem we obtain the desired bound. 
Corollary 3.7 Let $(X, L)$ be a polarized abelian surface of type $(1, d)$ with $\rho(X)=1$. Let $x$ be a point of $X$. Let $X_{m}$ be the subgroup of $m$-torsion points. Suppose that $\sqrt{2 d^{2}}$ is not an integer, then:

$$
\epsilon\left(L ; x+X_{m}\right)=2 \frac{d}{m^{2}} \frac{k_{0}}{l_{0}}=\sqrt{1-\frac{1}{l_{0}^{2}}} \sqrt{\frac{L^{2}}{m^{4}}}
$$

where $\left(l_{0}, k_{0}\right)$ is the primitive solution of Pell's equation $l^{2}-2 d k^{2}=1$.

Proof: Note that, in this case, the minor number $n$ such that $n L$ descends under $q$ is $n=m^{2}$. Now, it is sufficient to apply the Theorem 1.2

Remark 3.8 In [11], Tutaj-Gasinska obtains a bound for the Seshadri constant in half-periods of a line bundle on a polarized abelian surface of type $(1, d)$ :

$$
\epsilon\left(L ; X_{2}\right) \leq 2 \sqrt{1-\frac{1}{l_{0}^{2}}} \sqrt{\frac{L^{2}}{16}}
$$

where $\left(l_{0}, k_{0}\right)$ is the primitive solution of Pell's equation $l^{2}-32 d k^{2}=1$. Here, we see that we exact value appears when we use the Pell's equation $l^{2}-2 d k^{2}=1$.

Corollary 3.9 Let $(X, L)$ be a polarized abelian surface of type $(1, d)$ with $\rho(X)=1$. Let $e_{1}, e_{2}$ be two half periods of $X$. Suppose that $\sqrt{d}$ is not an integer.

1. If $e_{1}-e_{2} \in K(L)$ then

$$
\epsilon\left(L ; e_{1}, e_{2}\right)=d \frac{k_{0}}{l_{0}}
$$

where $\left(l_{0}, k_{0}\right)$ is the primitive solution of Pell's equation $l^{2}-d k_{0}^{2}=1$.

2. If $e_{1}-e_{2} \notin K(L)$ then

$$
\epsilon\left(L ; e_{1}, e_{2}\right)=2 d \frac{k_{0}}{l_{0}}
$$

where $\left(l_{0}, k_{0}\right)$ is the primitive solution of Pell's equation $l^{2}-4 d k_{0}^{2}=1$.

Proof: Consider the group $G=\left\langle e_{1}-e_{2}\right\rangle$. It has order two. Moreover, $L$ descends to a line bundle in $X / G$ if and only if $e_{1}-e_{2} \in K(L)$. Now we only have to apply the Theorem 1.2

Remark 3.10 In [12], a similar study is made. However, the Theorem (1, [12]) does not distinguish which are the two half-periods where the Seshadri constant is computed. The problem is the application of Lemma (19, [12]). It says that one can choose a line bundle $L_{c}$ such that any two half periods $e_{1}, e_{2}$ have the same parity. However, this changes the number of even and odd half periods of L. From this, the arguments on page (532, [12]) can fail. 
We could use similar arguments to compute the multiple Seshadri constants at points of a finite subgroup of line bundles on abelian surfaces with Picard number great than 1 . The simple Seshadri constants of these surfaces were computed by C. Schultz in 8. Anyway, we know that the simple Seshadri constant of a line bundle on any abelian surface is always rational. From this:

Theorem 3.11 Let $(X, L)$ be a polarized abelian surface. The multiple Seshadri constant of $L$ at the points of a finite subgroup is rational.

\section{References}

[1] BAUER, T. Seshadri constants and periods of polarized abelian varieties. Math. Ann. 312, 607-623 (1998).

[2] Bauer, T. Seshadri constants on algebraic surfaces. Math. Ann. 313, 547-583 (1999).

[3] Birkenhake, Ch.; Lange, H. Complex abelian vareties. Springer-Verlag (1992).

[4] Biran, P. Constructing new ample divisors out of old ones. Duke Math. J. 98 113-135, (1999).

[5] Demailly, J.-P. Singular hermitian metrics on positive line bundles Lecture Notes Math. 1507, 87-104 (1992).

[6] Harbourne, B. Seshadri constants and very ample divisors on algebraic surfaces. J. Reine Angew. Math 559, 115-122 (2003).

[7] Nakamaye, M.J. Seshadri constants and the geometry of surfaces. J. Reine Angew. Math. 564, 205-214 (2003).

[8] Schultz, Ch. Seshadri constants on abelian surfaces. Thesis, Marburg, 2004.

[9] Steffens, A. Remarks on Seshadri constants. Math. Z. 227, 505-510 (1998).

[10] Strycharz-Szemberg, B.; Szemberg, T. Remarks on the Nagata conjecture. Serdica Math. J. 30, N 2-3, 405-430 (2004)

[11] Tutaj-Gasińska, H. Seshadri constants in half-periods of an abelian surface. J. Pure Appl. Algebra 194, N1-2, 183-191 (2004).

[12] Tutaj-Gasińska, H. Seshadri constants in two half periods. Arch. Math. 85, N. 6, 514-526 (2005). 
E-mail: lfuentes@udc.es

Luis Fuentes García.

Departamento de Métodos Matemáticos y Representación.

E.T.S. de Ingenieros de Caminos, Canales y Puertos.

Universidad de A Coruña. Campus de Elviña. 15192 A Coruña (SPAIN) 